\title{
Logarithmic corrections in the free energy of monomer-dimer model on plane lattices with free boundaries
}

\author{
Yong Kong* \\ Department of Mathematics \\ National University of Singapore \\ Singapore 117543
}

(Dated: September 7, 2018)

\begin{abstract}
Using exact computations we study the classical hard-core monomer-dimer models on $m \times n$ plane lattice strips with free boundaries. For an arbitrary number $v$ of monomers (or vacancies), we found a logarithmic correction term in the finite-size correction of the free energy. The coefficient of the logarithmic correction term depends on the number of monomers present $(v)$ and the parity of the width $n$ of the lattice strip: the coefficient equals to $v$ when $n$ is odd, and $v / 2$ when $n$ is even. The results are generalizations of the previous results for a single monomer in an otherwise fully packed lattice of dimers.

PACS numbers: 05.50.+q, 02.10.De, 02.70.-c, 11.25.Hf
\end{abstract}

The monomer-dimer problem is a classical model in statistical physics. In the model the diatomic molecules are modeled as rigid dimers which occupies two adjacent sites in a regular lattice and no lattice site is covered by more than one dimer. The lattice sites that are not covered by the dimers are regarded as occupied by monomers. A central problem of the model is to enumerate the dimer configurations on the lattice. A breakthrough came in 1961 when an elegant exact closed-form solution was found for a special case of the model in plane lattices, namely when the lattice is completely covered by dimers (the close-packed dimer problem, or dimercovering problem) [1]. The method used to achieve this solution, however, cannot be extended to tackle the more general monomer-dimer problem where there are vacancies in the lattice, and the problem remains unsolved notwithstanding years of efforts. Rigorous results exists only for series expansion in low dimer density [2], lower bounds on free energy [3], monomer-monomer correlation function of two monomers in a lattice otherwise packed with dimers [4], and locations of zeros of partition functions [5]. Some approximate methods have been proposed [6]. One recent advance is an analytic solution to the special case of the problem where there is a single vacancy at certain specific sites on the boundary of the lattice [7].

There is renewed interest in monomer-dimer problem recently. The interest comes from different directions. Besides the intrinsic interest of the problem itself and its close relation to the well-studied Ising model [8], the model also acts as the classical limit of the recently introduced quantum dimer model [9], which has been investigated intensively as the central model in modern theories of strongly correlated quantum matter.

The problem also attracts attention in the field of

*Electronic address: matky@nus.edu.sg computational complexity. It has been shown that twodimensional monomer-dimer problem is computationally intractable and belongs to the "\#P-complete" class 10]. \#P-complete class plays the same role for counting problems (such as counting dimer configurations discussed here) as the more familiar NP-complete class for the decision problems (such as the well-known traveling salesman problem). The \#P-complete problems are at least as hard as the NP-complete problems 11. If any problem in the \#P-complete class is found to be solvable, every problem in \#P class is solvable. Currently it is not clear whether there exists any such solution to the \#Pcomplete or NP-complete class problems, and " $\mathrm{P}$ verse NP" problem is the perhaps the major outstanding problem in theoretical computer science.

In this Letter we report the logarithmic correction in the free energy of the monomer-dimer model with arbitrary number of monomers on plane lattice strips of size $m \times n$, where the width $n$ is fixed, and address the finding in the context of universality, scaling, and exact finitesize corrections in critical systems. These topics have been studied intensively in recent decades. As one of the few non-trivial exactly solved statistical lattice models, the dimer model attracts much attention recently to test the predictions of conformal field theory [12] and finitesize scaling [13]. Unlike other models, the (close-packed) dimer model on plane lattice shows certain peculiarity with respect to the predictions of the theories, both for the central charge [14, 15] and for the logarithmic corrections [14]. The free energy of a two-dimensional system at criticality is assumed to have the form of [12, 13]

$$
F_{m, n}=m n f_{b}+(m+n) f_{s}+C \ln m+D+o(1) .
$$

The first two terms, $f_{b}$ and $f_{s}$, are the "bulk" and "surface" terms, respectively. These two terms are not universal and depend on the details of the model. The other two coefficients, $C$ and $D$, are supposed to be universal, depending only on the shape of the system and the boundary conditions. The dimensionless coefficient $D$ is 
related to the central charge of the system and the boundary conditions in the transversal direction [12]. The coefficient of the logarithmic term $C$ depends on the geometry of the system. For systems with corners, as for the lattices with free boundaries discussed here, $C=\sum_{i} u_{i}$, with some universal contribution $u_{i}$ from each corner [13].

From the exact solution of the close-packed dimer problem, the asymptotic expression of the free energy $F_{m, n}=\ln a_{m n / 2}$ was found to be [16]

$$
F_{m, n}=m n f_{b}+(m+n) f_{s}+D_{n}[(m+1) /(n+1)]+o\left(n^{-2+\delta}\right)
$$

where $\delta>0$ and $D_{n}$ depends on the geometry of the lattice and the parity of $n$. Two issues arise from this asymptotic expression. The first issue is related to the central charge, because the expansion depends on the parity of $n$ :

$$
\frac{F_{m, n}}{m n}=\frac{G}{\pi}+\left[\frac{G}{\pi}-\frac{1}{2} \ln (1+\sqrt{2})\right] \frac{1}{n}+\frac{\pi}{24} \frac{1}{n^{2}}+\cdots
$$

when $n$ is even, and

$$
\frac{F_{m, n}}{m n}=\frac{G}{\pi}+\left[\frac{G}{\pi}-\frac{1}{2} \ln (1+\sqrt{2})\right] \frac{1}{n}-\frac{\pi}{12} \frac{1}{n^{2}}+\cdots
$$

when $n$ is odd. Here $G$ is the Catalan's constant. At the first look, the coefficients of $n^{-2}$, which are related to the central charge of the conformal field theory, are different for even and odd $n$, leading to $c=1$ for the even $n$ and $c=-2$ for odd $n$. Recently this issue has been discussed using logarithmic conformal field theory [15, 17]. It is pointed out that the coefficients of $n^{-2}$ are actually related to the effective central charge $c_{\text {eff }}=c-24 h_{\text {min }}$, where $h_{\min }$ is the conformal weight of the ground state and is a boundary dependent quantity. By using a bijection of the dimer coverings with a spanning tree and Abelian sandpile model, it is found that changing the parity of $n$ results in the change of the boundary conditions of the mapped spanning trees, leading to different $c_{\text {efff }}$. The central charge itself, however, remains unchanged $c=-2$ 15].

The second issue, which was pointed out previously 14], is the absence of corner contribution term predicted by the theory (Eq. 11) in the expansion of Eq. (2) of the close-packed dimer model. This issue, however, changes its nature when we look at the general monomerdimer model. Recently we found that when there is one monomer in the lattices, there is indeed a logarithmic term in the finite-size correction of the free energy [18]. In the following we generalize the results to plane lattices with arbitrary number of monomers, and found that the coefficients of the logarithmic correction term depend not only on the number of monomers, but also on the parity of the width of the lattice strip $n$.

The configurational grand canonical partition function of the system is

$$
Z_{m, n}(x)=a_{N} x^{N}+a_{N-1} x^{N-1}+\cdots+a_{0}
$$

where $a_{k}$ is the number of ways to arrange $k$ dimers on the $m \times n$ plane lattice with free boundaries. We are interested in the coefficients of fixed number $v$ of monomers, that is, $a_{(m n-v) / 2}$, when the width of the lattice strip $n$ is fixed and the length of the strip $m$ changes. When the width of the lattice strip $n$ is even, $v$ can only take even numbers; when $n$ is odd, $v$ can take either even or odd values based on the parity of $m$.

The full partition function have been calculated exactly for $n$ from 1 to 16 , using the method discussed in Refs. 18, 19]. The coefficients $a_{(m n-v) / 2}$ of the partition functions are extracted to fit the following function:

$\frac{\ln a_{(m n-v) / 2}}{m n}=c_{0}+\frac{c_{1}}{m}+\frac{c_{2}}{m^{2}}+\frac{c_{3}}{m^{3}}+\frac{c_{4}}{m^{4}}+\frac{b v l}{n} \frac{\ln (m+1)}{m}$.

where $b=1$ when $n$ is odd, and $b=1 / 2$ when $n$ is even. The reason to choose $\ln (m+1)$ instead of $\ln (m)$ is discussed in Ref. [18], and the choice will not affect the results discussed below.

For the close-packed dimer problem (where $v=0$ and $m n$ is even), the exact solution gives $c_{0}$ in Eq. (3) as [1]

$$
c_{0}^{e}(n)=\frac{1}{n} \ln \left[\prod_{l=1}^{\frac{n}{2}}\left(\cos \frac{l \pi}{n+1}+\left(1+\cos ^{2} \frac{l \pi}{n+1}\right)^{\frac{1}{2}}\right)\right] .
$$

For a given $n$, this exact expression of $c_{0}$ actually holds for any finite number $v$ of monomers. This was confirmed by fitting the data with $c_{0}$ as a free parameters. In the following we use $c_{0}^{e}(n)$ given in Eq. (4) for $c_{0}$. We fit the data extracted from the full partition functions to Eq. (3) for $v=0, \ldots, 12$ for odd $n$, and for $v=0,2,4, \ldots, 24$ for even $n$. The fitting results for $l$ are shown in Tables [1 and [II respectively [20]. As we did previously [18], in these fittings, only $m \geq m_{0}=100$ are used. The curves of fitting for two values of $n, n=11$ and $n=12$, are shown in Figure 1 and Figure 2 respectively. The fitting results for all the parameters when $n=11$ and $n=12$ are shown in Tables [II and IV respectively. These results lead clearly to the conclusion that for a fixed number of monomers $v$, there is a logarithmic correction term in the free energy $(m n)^{-1} \ln a_{(m n-v) / 2}$, and the coefficient of this term not only depends on $v$, but also on the parity of $n$, the width of the lattice strip: when $n$ is odd, the coefficient equals to $v$; when $n$ is even, the coefficient is $v / 2$. The lack of the logarithmic correction term found in the close-packed dimer problem $(v=0)$ 1, 14, 16] and the logarithmic correction term found in the oddby-odd lattice with one single vacancy $(v=1)[18$ are just special cases of this general result.

The close-packed dimer model, which is a special case of the general monomer-dimer model discussed here, already demonstrates intriguing connections with the theory of university and finite scaling [14, 15]. It would be interesting to see how the results reported here incorporate into the bigger picture. 
TABLE I: Coefficient of the logarithmic term $l$ from fitting $(m n)^{-1} \ln a_{(m n-v) / 2}$ to Eq. 3 for odd values of $n$, with $b=1$. Only data with $m \geq m_{0}=100$ are used in the fitting. For $n=1, m \leq 2000$; for $n=5, \ldots 13, m \leq 500$; for $n=15, m \leq 200$. Numbers in square brackets denote powers of 10 .

\begin{tabular}{|c|c|c|c|c|c|c|c|c|}
\hline \multirow[b]{2}{*}{$v$} & \multicolumn{8}{|c|}{$\mathrm{n}$} \\
\hline & 1 & 3 & 5 & 7 & 9 & 11 & 13 & 15 \\
\hline 0 & 0 & $-1.42355[-12]$ & $6.9891[-10]$ & $7.10235[-10]$ & $1.03087[-9]$ & $1.56222[-9]$ & $3.76785[-9]$ & $3.42299[-9]$ \\
\hline 1 & 1 & 1 & 1 & 1 & 1 & 1 & 1 & 1 \\
\hline 2 & 1 & 1 & 1 & 0.999999 & 0.999998 & 0.999997 & 0.999993 & 0.999968 \\
\hline 3 & 1 & 0.999998 & 0.999991 & 0.999975 & 0.999945 & 0.999897 & 0.999781 & 0.999088 \\
\hline 4 & 1 & 0.999988 & 0.999945 & 0.999851 & 0.99969 & 0.999449 & 0.998907 & 0.996177 \\
\hline 5 & 1 & 0.999962 & 0.999835 & 0.999576 & 0.999163 & 0.998585 & 0.99736 & 0.991937 \\
\hline 6 & 1 & 0.999912 & 0.999632 & 0.999108 & 0.998322 & 0.997282 & 0.995194 & 0.986896 \\
\hline 7 & 1 & 0.999836 & 0.999351 & 0.998494 & 0.997273 & 0.995726 & 0.99273 & 0.981618 \\
\hline 8 & 1 & 0.999721 & 0.998951 & 0.997671 & 0.995927 & 0.993799 & 0.98982 & 0.976008 \\
\hline 9 & 1 & 0.999581 & 0.998493 & 0.996767 & 0.9945 & 0.991812 & 0.986905 & 0.970599 \\
\hline 10 & 1 & 0.99939 & 0.997902 & 0.995649 & 0.99279 & 0.989492 & 0.983621 & 0.965005 \\
\hline 11 & 1 & 0.999181 & 0.997289 & 0.994527 & 0.991117 & 0.987267 & 0.980527 & 0.959804 \\
\hline 12 & 0.999999 & 0.998909 & 0.996529 & 0.99318 & 0.989159 & 0.984713 & 0.977081 & 0.954453 \\
\hline
\end{tabular}

TABLE II: Coefficient of the logarithmic term $l$ from fitting $(m n)^{-1} \ln a_{(m n-v) / 2}$ to Eq. B for even values of $n$, with $b=1 / 2$. Only data with $m \geq m_{0}=100$ are used in the fitting. For $n=2, m \leq 2000$; for $n=4, m \leq 1000$, for $n=6, \ldots 14, m \leq 500$; for $n=16, m \leq 130$. Numbers in square brackets denote powers of 10 .

\begin{tabular}{|c|c|c|c|c|c|c|c|c|}
\hline \multirow[b]{2}{*}{$v$} & \multicolumn{8}{|c|}{$\mathrm{n}$} \\
\hline & 2 & 4 & 6 & 8 & 10 & 12 & 14 & 16 \\
\hline 0 & $1.21643[-11]$ & $1.3329[-10]$ & $5.89405[-10]$ & $9.28482[-10]$ & $9.0419[-10]$ & $1.13781[-9]$ & $8.97751[-8]$ & $9.45733[-5]$ \\
\hline 2 & 1 & 1 & 1 & 0.999999 & 0.999999 & 1 & 1.00017 & 1.00218 \\
\hline 4 & 1 & 1 & 1.00001 & 1.00002 & 1.00006 & 1.00012 & 1.00075 & 1.00026 \\
\hline 6 & 1 & 1 & 1.00002 & 1.00003 & 1.00004 & 1 & 0.998332 & 0.990178 \\
\hline 8 & 1 & 1 & 0.999994 & 0.999931 & 0.999739 & 0.999318 & 0.993311 & 0.98074 \\
\hline 10 & 1 & 0.999997 & 0.999907 & 0.999639 & 0.999043 & 0.998001 & 0.987848 & 0.977344 \\
\hline 12 & 1 & 0.999982 & 0.999718 & 0.999103 & 0.997943 & 0.996201 & 0.983945 & 0.981147 \\
\hline 14 & 1 & 0.999951 & 0.9994 & 0.998314 & 0.996526 & 0.994189 & 0.982769 & 0.991167 \\
\hline 16 & 1 & 0.999897 & 0.998938 & 0.997305 & 0.994942 & 0.99226 & 0.984712 & 1.00579 \\
\hline 18 & 0.999997 & 0.999816 & 0.998332 & 0.996139 & 0.993357 & 0.990678 & 0.989654 & 1.02348 \\
\hline 20 & 0.999992 & 0.999702 & 0.997596 & 0.994896 & 0.991933 & 0.989648 & 0.997209 & 1.04299 \\
\hline 22 & 0.999983 & 0.999551 & 0.996756 & 0.993664 & 0.990808 & 0.989309 & 1.00689 & 1.06337 \\
\hline 24 & 0.99997 & 0.999361 & 0.995843 & 0.992527 & 0.990091 & 0.989743 & 1.01822 & 1.08398 \\
\hline
\end{tabular}

TABLE III: Fitting $(m n)^{-1} \ln a_{(m n-v) / 2}$ to Eq. [3] for $n=11$ with $b=1$ and $c_{0}$ fixed as $c_{0}^{e}(11)=0.276016$ given by Eq. 4 Only data with $m \geq m_{0}=100$ are used in the fitting. Numbers in square brackets denote powers of 10 .

\begin{tabular}{cccccc}
\hline \hline$v$ & $c_{1}$ & $c_{2}$ & $c_{3}$ & $c_{4}$ & $l$ \\
\hline 0 & -0.125966 & $8.75546[-8]$ & $-8.24421[-6]$ & 0.000319302 & $1.56222[-9]$ \\
1 & -0.0561077 & -0.140372 & 0.0319911 & -0.0345653 & 1 \\
2 & -0.0492583 & 0.474695 & -4.7249 & 23.4862 & 0.999997 \\
3 & -0.0790665 & 1.82165 & -36.8528 & 449.079 & 0.999897 \\
4 & -0.133937 & 3.79131 & -103.787 & 1715.28 & 0.999449 \\
5 & -0.207035 & 6.24906 & -203.292 & 3861.49 & 0.998585 \\
6 & -0.293685 & 9.05639 & -328.77 & 6728.3 & 0.997282 \\
7 & -0.391277 & 12.162 & -480.3 & 10421.5 & 0.995726 \\
8 & -0.496769 & 15.4114 & -645.37 & 14479.9 & 0.993799 \\
9 & -0.609818 & 18.8669 & -833.317 & 19374.1 & 0.991812 \\
10 & -0.727328 & 22.305 & -1022.43 & 24212.7 & 0.989492 \\
11 & -0.850844 & 25.9246 & -1235.21 & 30014.7 & 0.987267 \\
12 & -0.976389 & 29.3893 & -1437.02 & 35307.6 & 0.984713 \\
\hline \hline
\end{tabular}

[1] P. W. Kasteleyn, Physica 27, 1209 (1961); H. N. V. Temperley and M. E. Fisher, Philos. Mag. 6, 1061 (1961); M. E. Fisher, Phys. Rev. 124, 1664 (1961).

[2] J. F. Nagle, Phys. Rev. 152, 190 (1966); D. Gaunt, Phys. Rev. 179, 174 (1969).

[3] J. Bondy and D. Welsh, Proc. Camb. Phil. Soc. Math.
Phys. Sci. 62, 503 (1966); J. Hammersley, Proc. Camb. Phil. Soc. Math. Phys. Sci. 64, 455 (1968); J. Hammersley and V. Menon, J. Inst. Math. Appl. 6, 341 (1970).

[4] M. E. Fisher and J. Stephenson, Phys. Rev. 132, 1411 (1963); R. E. Hartwig, J. Math. Phys. 7, 286 (1966).

[5] O. J. Heilmann and E. H. Lieb, Commun. Math. Phys. 


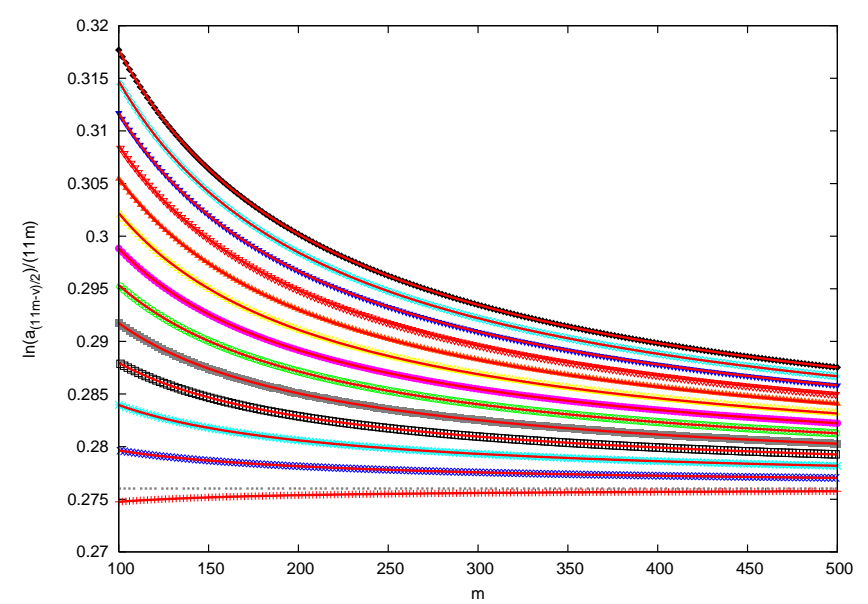

FIG. 1: (Color online) The original data of $(m n)^{-1} \ln \left(a_{(m n-v) / 2}\right)$ and the fitted curves for $n=11$ and $v=0,1, \ldots, 12$. The curve for $v=0$ is on the bottom and that for $v=12$ is on the top. The dashed horizontal line is $c_{0}^{e}(11)=0.276016$ from exact expression Eq. 4

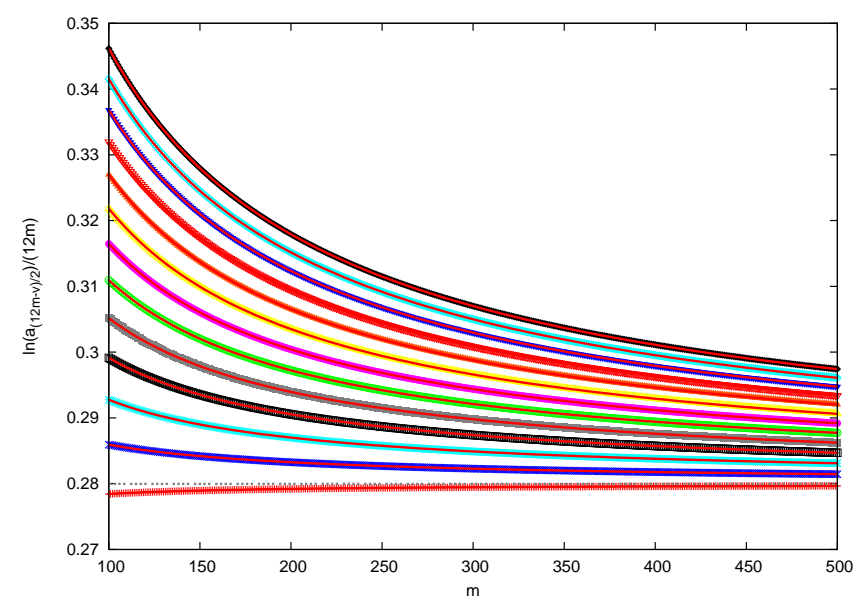

FIG. 2: (Color online) The original data of $(m n)^{-1} \ln \left(a_{(m n-v) / 2}\right)$ and the fitted curves for $n=12$ and $v=0,2, \ldots, 24$. The curve for $v=0$ is on the bottom and that for $v=24$ is on the top. The dashed horizontal line is $c_{0}^{e}(12)=0.279976$ from exact expression Eq. 4

TABLE IV: Fitting $(m n)^{-1} \ln a_{(m n-v) / 2}$ to Eq. 3 for $n=12$ with $b=1 / 2$ and $c_{0}$ fixed as $c_{0}^{e}(12)=0.279976$ given by Eq. 4 Only data with $m \geq m_{0}=100$ are used in the fitting. Numbers in square brackets denote powers of 10 .

\begin{tabular}{cccccc}
\hline \hline$v$ & $c_{1}$ & $c_{2}$ & $c_{3}$ & $c_{4}$ & $l$ \\
\hline 0 & -0.154113 & $5.91813[-8]$ & $-5.64565[-6]$ & 0.000221538 & $1.13781[-9]$ \\
2 & 0.219858 & -0.748376 & -2.59592 & -16.0843 & 1 \\
4 & 0.535917 & -2.62009 & -9.74674 & 325.144 & 1.00012 \\
6 & 0.818481 & -5.66056 & -12.3135 & 1291.39 & 1 \\
8 & 1.07862 & -10.0074 & 12.3026 & 2471.16 & 0.999318 \\
10 & 1.32299 & -15.8166 & 92.4989 & 2982.18 & 0.998001 \\
12 & 1.55565 & -23.1898 & 253.569 & 1852.54 & 0.996201 \\
14 & 1.77871 & -32.1427 & 512.746 & -1715.1 & 0.994189 \\
16 & 1.99286 & -42.6063 & 878.094 & -8235.31 & 0.99226 \\
18 & 2.19782 & -54.4469 & 1349.67 & -17937.8 & 0.990678 \\
20 & 2.39273 & -67.4891 & 1921.53 & -30816.5 & 0.989648 \\
22 & 2.57645 & -81.5376 & 2583.82 & -46694.5 & 0.989309 \\
24 & 2.74778 & -96.3937 & 3324.52 & -65284.5 & 0.989743 \\
\hline \hline
\end{tabular}

25, 190 (1972); C. Gruber and H. Kunz, Commun. Math. Phys. 22, 133 (1971).

[6] C. Kenyon, D. Randall, and A. Sinclair, J. Stat. Phys. 83, 637 (1996).

[7] W.-J. Tzeng and F. Y. Wu, J. Stat. Phys. 110, 671 (2003).

[8] P. W. Kasteleyn, J. Math. Phys. 4, 287 (1963); M. E. Fisher, J. Math. Phys. 7, 1776 (1966).

[9] D. S. Rokhsar and S. A. Kivelson, Phys. Rev. Lett. 61, 2376 (1988); F. Alet, J. L. Jacobsen, G. Misguich, V. Pasquier, F. Mila, and M. Troyer, Phys. Rev. Lett. 94, 235702 (2005).

[10] M. Jerrum, J. Stat. Phys. 48, 121 (1987); 59, 1087(E) (1990).

[11] M. R. Garey and D. S. Johnson, Computers and Intractability, A Guide to the Theory of NP-Completeness (W.H. Freeman and Company, New York, 1979).

[12] H. W. J. Blöte, J. L. Cardy, and M. P. Nightingale, Phys. Rev. Lett. 56, 742 (1986); I. Affleck, Phys. Rev. Lett. 56, 746 (1986).

[13] J. L. Cardy and I. Peschel, Nucl. Phys. B300[FS22], 377 (1988); V. Privman, Phys. Rev. B 38, 9261 (1988).

[14] J. G. Brankov, J. Math. Phys. 36, 5071 (1995).

[15] N. S. Izmailian, V. B. Priezzhev, P. Ruelle, and C.-K. $\mathrm{Hu}$, Phys. Rev. Lett. 95, 260602 (2005).

[16] A. E. Ferdinand, J. Math. Phys. 8, 2332 (1967).

[17] C. Itzykson, H. Saleur, and J.-B. Zuber, Europhys. Lett. 2, 91 (1986).

[18] Y. Kong, Phys. Rev. E 73, 016106 (2006).

[19] Y. Kong, J. Chem. Phys. 111, 4790 (1999).

[20] Exact result exists for $n=1: a_{(m-v) / 2}=\left(\begin{array}{c}(m+v) / 2 \\ v\end{array}\right)=$ $\left(2^{v} v !\right)^{-1}(m+v)(m+v-2) \cdots(m-v+2)$, which gives $l=1$ exactly. The fitting results as listed in Table \are used as a check for the fitting procedure. 